\title{
Serum fetuin-A and recurrent urolithiasis in young adults
}

\author{
Domenico Prezioso $^{1}$, Alberto Saita ${ }^{2}$, Mario Motta ${ }^{2}$, Massimo Porena ${ }^{3}$, \\ Carla Micheli ${ }^{3}$, Ester Illiano ${ }^{1}$, Dario Bruzzese ${ }^{4}$, Vincenzo Bisesti ${ }^{5}$, Paolo Ferrari ${ }^{6}$, \\ Tullio Lotti $^{7}$, Domenico Russo ${ }^{5}$ \\ ${ }^{1}$ Department of Gynecology, Obstetric and Urology, University Federico II, Naples, Italy; \\ 2 Department of Urology, "Ospedale Vittorio Emanuele", University of Catania, Catania, Italy; \\ ${ }^{3}$ Department of Urology and Andrology, Ospedale Santa Maria della Misericordia, University of Perugia, \\ Perugia, Italy; \\ ${ }^{4}$ Department of Preventive Medical Sciences, University Federico II, Naples, Italy; \\ ${ }^{5}$ Department of Nephrology, University Federico II, Naples Italy; \\ ${ }^{6}$ Department of Urology, Ospedale Hesperia, Modena, Italy; \\ ${ }^{7}$ University Federico II, Naples, Italy.
}

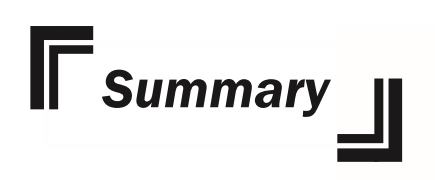

Objective: Recurrence of urolithiasis is frequent. There are no reliable markers able to indicate recurrent stone former patients. Fetuin-A inhibits hydroxyapatite crystals formation and expansion. This study aims at evaluating whether serum fetuin-A may predict recurrent urolithiasis in young adults.

Materials and methods: This is a multicentre study. Young adults patients with recurrent urolithiasis attending 3 urology clinics were enrolled from July 2011 to December 2012. Inclusion criteria were: age 18-40 years, presence of more than one kidney stone. Exclusion criteria were: diabetes mellitus, metabolic disorders, obesity, hypertension, cardiovascular disease, infection diseases. Controls were participants without history of urolithiasis and currently undetected stones. Routine biochemistry, serum concentration of oxalate, fetuin- $A$, and parathyroid hormone (PTH) were assessed; $24 / \mathrm{h}$ urinary excretion of creatinine, uric acid, calcium, sodium, phosphorus, potassium, magnesium, glucose, oxalate, amylase, and protein was measured. Kidney ultrasonography and plain X-ray examination was performed.

Results: The total cohort was represented by 120 young adults participants (90 patients, and 30 controls). Clinical characteristics were not different between patients and controls. No significant differences were found in serum concentrations as well as in 24/h urinary excretion of recorded variables. No significant difference was found in serum concentration of fetuin-A (median $35.1 \pm$ 18.62 SD Vs $35.12 \pm 14.12, \mu g / m l ; p=0,908$ ).

Conclusions: The data of present study do not substantiate the hypothesis that serum fetuin-A may be a reliable predictor of recurrent urolithiasis in young adults.

KEY WORDS: Calcification; Inhibition; Extra osseous calcification; Fetuin-A; Recurrent urolithiasis.

\section{INTRODUCTION}

Urolithiasis represents a process of unwanted calcification and the recurrence is the rule (1). The underlying mechanisms of stone formation are not clearly known. In addition, there are no markers able to distinguish between patients who will have stone recurrence from those who will not. Therefore, it is clinically important to identify potential factor(s) that may help clinicians in making decision.
Along with the multiple traditional pathogenic factors involved in stone formation (1-3), great relevance has been recently given to inhibitors of ectopic calcification $(4,5)$. Inhibitors raise the concentrations of calcium and oxalate required for spontaneous formation of new crystals and decrease crystal growth, aggregation, and binding to renal cells.

Fetuin-A plays a crucial role in the extra osseous calcifi- 
cation process by inhibiting the formation and expansion of hydroxyapatite crystals (6-8). Unfortunately there is scarce information on fetuin-A in patients with recurrent urolithiasis. One sole study evaluated urine fetuin concentration in patients with urolithiasis (9). Since urinary excretion of fetuin-A may result inaccurate as ascertained in several renal injury models $(10,11)$, in the present study serum fetuin-A was assessed in patients with recurrent multiple urolithiasis and compared with that of normal subjects. So far there is no data on this issue.

\section{Materials AND METHOdS}

This is a multicentre study performed in outpatients attending 3 urology clinics from July 2011 to December 2012. The study protocol was approved by the institutional review board of Catania. Procedures were carried out according to the declaration of Helsinki. Before enrollment all patients signed informed consent.

Inclusion criteria were: age 18-40 years, recurrent urolithiasis, current presence of kidney stones. Exclusion criteria were: diabetes mellitus, metabolic disorders, congenital or acquired dyslipidemia, obesity, hypertension, cardiovascular disease, infection diseases.

Serum concentration of glucose, creatinine, sodium, potassium, uric acid, calcium, phosphorus, magnesium, total cholesterol, triglycerides, oxalate, fetuin-A, and parathyroid hormone (PTH) was assayed.

Urinary excretion of creatinine, uric acid, calcium, sodi- um, phosphorus, potassium, magnesium, glucose, oxalate, amylase, protein was assessed on 24-hour collections. Kidney ultrasonography and plain X-ray examination was performed.

Serum fetuin-A was measured by Human Fetuin-A ELISA (Biovendor Laboratory Medicine). The intra- and interassay variations were evaluated by measuring three different samples in 33 replicates (intra-assay coefficient of variation $<5,2 \%$, inter-assay coefficient of variation $<3,8 \%$, limit of detection $0.35 \mathrm{mg} / \mathrm{L}$ ).

The body mass index (BMI) of patients and controls was calculated.

Mann-Whitney test (abnormal data distribution), nonpaired t-test (normal data distribution), chi square, and Fisher exact test were used to compare data of subjects with and without urolithiasis; $\mathrm{P}<0.05$ was considered as significant.

\section{RESULTS}

We enrolled 90 young adults patients (30 patients in each centre; 58 men, 32 women) with documented recurrent formation of urinary stones, and 30 individuals (10 in each centre; 14 men, 16 women) without urolithiasis.

Clinical characteristics and laboratory variables of patients with and without urolithiasis are reported in Tables 1, 2 and 3. Both groups were homogeneous for age, and BMI. There were no significant differences in serum concentration except for serum calcium and phosphorus; both ions

Table 1.

Clinical characteristics of controls and patients with urolithiasis.

\begin{tabular}{|c|c|c|c|c|c|}
\hline & \multicolumn{2}{|c|}{ Controls } & \multicolumn{2}{|c|}{ Patients } & \multirow[t]{2}{*}{$\mathbf{p}$} \\
\hline & Mean \pm SD & Median (range) & Mean \pm SD & Median (range) & \\
\hline Age & $28.47 \pm 5.75$ & 27.5 [19-40] & $31.21 \pm 7.18$ & 32 [18-42] & 0.068 \\
\hline BMI & $23.47 \pm 3.06$ & 24 [18-31] & $23.03 \pm 2.73$ & 23 [18-30] & 0.413 \\
\hline
\end{tabular}

BMI: body mass index.

Table 2.

Baseline serum concentration of variables measured in controls and patients with urolithiasis.

\begin{tabular}{|l|c|c|c|}
\hline & Controls & Patients & Mean \pm SD \\
\hline Variables & Mean \pm SD & p \\
\hline Fetuin-A $(\mu \mathrm{g} / \mathrm{ml})$ & $35.1 \pm 18.6(35.1 \pm 18.6 \mu \mathrm{g} / \mathrm{ml})$ & $35.1 \pm 14.1(35.1 \pm 14.1 \mu \mathrm{g} / \mathrm{ml})$ & 0.908 \\
\hline Oxalate $(\mu \mathrm{mol} / \mathrm{L})$ & $97 \pm 22(8.7 \pm 1.9 \mathrm{mg} / \mathrm{L})$ & $124 \pm 73(11.1 \pm 6.6 \mathrm{mg} / \mathrm{L})$ & 0.612 \\
\hline PTH $(\mathrm{ng} / \mathrm{L})$ & $46 \pm 20(46 \pm 20 \mathrm{pg} / \mathrm{mL})$ & $47 \pm 18(47 \pm 18 \mathrm{pg} / \mathrm{mL})$ & 0.701 \\
\hline Sodium $(\mathrm{mmol} / \mathrm{L})$ & $144 \pm 3.4(144 \pm 3.4 \mathrm{mEq} / \mathrm{L})$ & $142 \pm 2.2(142 \pm 2.2 \mathrm{mEq} / \mathrm{L})$ & 0.177 \\
\hline Potassium $(\mathrm{mmol} / \mathrm{L})$ & $4.6 \pm 0.9(4.6 \pm 0.9 \mathrm{mEq} / \mathrm{L})$ & $4.7 \pm 0.4(4.7 \pm 0.4 \mathrm{mEq} / \mathrm{L})$ & 0.028 \\
\hline Calcium $(\mathrm{mmol} / \mathrm{L})$ & $2.3 \pm 0.10(9.22 \pm 0.42 \mathrm{mg} / \mathrm{dL})$ & $2.4 \pm 0.10(9.56 \pm 0.42 \mathrm{mg} / \mathrm{dL})$ & 0.041 \\
\hline Phosphorus $(\mathrm{mmol} / \mathrm{L})$ & $1.29 \pm 0.20(4.0 \pm 0.62 \mathrm{mg} / \mathrm{dL})$ & $1.15 \pm 0.29(3.57 \pm 0.89 \mathrm{mg} / \mathrm{dL}$ & 0.028 \\
\hline Total cholesterol $(\mathrm{mmol} / \mathrm{L})$ & $4.65 \pm 0.75(181 \pm 29 \mathrm{mg} / \mathrm{dL})$ & $4.78 \pm 1.16(187 \pm 45 \mathrm{mg} / \mathrm{dL})$ & 0.331 \\
\hline Triglycerides $(\mathrm{mmol} / \mathrm{L})$ & $0.85 \pm 0.21(75 \pm 19 \mathrm{mg} / \mathrm{dL})$ & $1.06 \pm 0.55(94 \pm 49)$ & 0.396 \\
\hline
\end{tabular}

PTH: parathyroid hormone. 
Table 3.

$24 / h$ urinary excretion of variables measured in controls and patients with urolithiasis.

\begin{tabular}{|l|c|c|c|}
\hline Phosphate $(\mathrm{mmol} / 24 \mathrm{~h})$ & Controls & Patients & $334 \pm 108(1037 \pm 335 \mathrm{mg})$ \\
\hline Uric acid $(\mathrm{\mu mol} / 24 \mathrm{~h}$ & $367 \pm 68(1141 \pm 211 \mathrm{mg})$ & 0.451 \\
\hline Calcium $(\mathrm{mmol} / 24 \mathrm{~h})$ & $37.4 \pm 9.2(150 \pm 37 \mathrm{mg})$ & $59.6 \pm 34.9(239 \pm 140 \mathrm{mg})$ & 0.095 \\
\hline Amylase $(\mathrm{U} / 24 \mathrm{~h})$ & $236 \pm 171.42$ & $247.77 \pm 100.5$ & 0.158 \\
\hline Sodium $(\mathrm{mmol} / 24 \mathrm{~h})$ & $164.14 \pm 36.06$ & $179.33 \pm 61.58$ & 0.315 \\
\hline Potassium $(\mathrm{mmol} / 24 \mathrm{~h})$ & $73.67 \pm 22.36$ & $57.29 \pm 21.6$ & 0.058 \\
\hline Chlorine $(\mathrm{mmol} / 24 \mathrm{~h})$ & $182.7 \pm 46.6$ & $186 \pm 69.97$ & 0.747 \\
\hline Proteinuria $(\mathrm{mg} / 24 \mathrm{~h})$ & $142 \pm 42$ & $188 \pm 134$ & 0.508 \\
\hline Magnesium $(\mathrm{mmol} / 24 \mathrm{~h})$ & $58 \pm 25.1(141 \pm 61 \mathrm{mg})$ & $44.4 \pm 17.8(108 \pm 43 \mathrm{mg})$ & 0.148 \\
\hline Glycosuria $(\mathrm{mmol} / 24 \mathrm{~h})$ & $4.8 \pm 1.5(87 \pm 27 \mathrm{mg})$ & $4.7 \pm 1.9(85 \pm 35 \mathrm{mg})$ & 0.771 \\
\hline
\end{tabular}

were significantly different between patients with and without urolithiasis (serum calcium: mean $9.22 \mathrm{mg} / \mathrm{dL} \pm$ $0.42 \mathrm{SD}$ vs $9.56 \mathrm{mg} / \mathrm{dL} \pm 0.42 \mathrm{SD} ; \mathrm{p} 0.041$; serum phosphorus: mean $4.0 \mathrm{mg} / \mathrm{dL} \pm 0.62 \mathrm{SD}$ vs $3.57 \mathrm{mg} / \mathrm{dL} \pm 0.89$ SD; $p$ 0.028). There were no significant differences in $24 / \mathrm{h}$ urinary excretion of measured variables. Serum concentration of fetuin was similar in patients and controls (mean $35.1 \pm 18.62$ SD vs $35.12 \pm 14.12 \mu \mathrm{g} / \mathrm{ml} ; \mathrm{p}=0.908)$.

\section{Discussion}

In this study a potential association was investigated between serum concentration of fetuin-A and recurrent urolithiasis in young adults.

Formation of stones within the urinary tract is a complex process driven by multiple factors. Although normal urine is frequently supersaturated with respect to calcium and oxalate, most individuals do not form stones. Typically, any crystals formed are rapidly passed before achieving a size sufficient for retention. Increased quantities of calcium and/or oxalate are excreted by many stone former. Therefore, increased supersaturation alone does not account for urinary stone; as a consequence, other factors may influence their formation and growth. Therefore, it is clinically relevant to identify new potential factor(s) that may help clinicians in making decision. Fetuin-A inhibits the precipitation of hydroxyapatite from supersaturated solutions of calcium and phosphate by forming fetuin-mineral complex, a high molecular mass complex $(6-8,12)$. This inhibitory action is at least in part facilitated by the transient formation of soluble, colloidal spheres, so-called calciprotein particles, containing fetuin$\mathrm{A}$, calcium, and phosphate. The action of fetuin-A is most prominent in organs involved in the secretion or transport of mineral-rich fluids or in the generation of local $\mathrm{pH}$ changes such as the kidney. Fetuin-A deficient rats have calcification in the pelvis but not in the medulla or the cortex compared to wild type; in this experimental model, calcification was primarily a consequence of the lack of fetuin-A and not of renal damage. Interestingly, animals had calcium and phosphate concentrations within the normal range; this finding rules out hypocalcaemia or hyperphosphatemia as a cause of the ectopic calcification. Therefore, the underlying mechanism may involve a direct interaction of fetuin-A with the mineral phase and the prevention of large crystal formation.

Owing to the crucial role of fetuin-A in the process leading to extra osseous calcification, it was stimulating to evaluate its potential role in patients with recurrent urolithiasis.

Ascertaining a role of serum fetuin A in recurrent urolithiasis should be of clinical interest considering that the recurrence of urolithiasis is the rule in all formingstone patients and that there are no laboratory markers able to distinguish between patients who will have stone recurrence from those who will not.

There is at present scarce information on urine fetuin-A in patients with recurrent urolithiasis. In one sole study, urine fetuin concentration was found lower in 38 patients with urolithiasis who were compared to 22 controls (9). The lower urine fetuin-A levels were not due to other conventional promoters and inhibitors of urine crystallization. On the basis of these data, measurement of urinary fetuinA was suggested as more reliable risk predictor than the traditional markers of recurrent urolithiasis (9).

This interesting suggestion, however, should be viewed with caution taking in account the data attained by proteonomics that have shown that urinary excretion of fetuin-A is altered in several renal injury models (11). Urinary exosomal fetuin-A is elevated in patients with acute kidney injury in intensive care unit (11). Recently, urinary exosomal fetuin-A levels was found significantly increased after cisplatin-induced tubule damage (11). Thus, urinary fetuin-A excretion may be strongly affected in presence of structural renal injury.

The data of the present study do not confirm the hypothesized relationships between serum fetuin-A concentration and recurrent urolithiasis. In fact, there was no significant difference in serum fetuin-A concentration between patients and controls. Similarly, there were no significant differences in traditional variables. These data confirm that there are no markers able to distinguish between patients who will have stone recurrence from 
those who will not. In addition, neither serum concentration nor urinary excretion of fetuin-A may be regarded as reliable predictors of recurrent urolithiasis. Hopefully, studies performed with proteonomics may find true predictor(s) of recurrent urolithiasis.

\section{Conclusions}

The data of present study do not substantiate the hypothesis that serum fetuin-A may be a reliable predictor of recurrent urolithiasis in young adults.

\section{References}

1. Coe FL, Favus MJ, Asplin JR. Nephrolithiasis. In Brenner BM, ed. The Kidney. $7^{\text {th }}$ ed, Philadelphia:Saunders. 2004; 1819-1866.

2. De Yoreo JJ, Qiu SR, Hoyer JR. Molecular modulation of calcium oxalate crystallization. Am J Physiol Renal Physiol. 2006; 291: F1123-F1132,

3. Asplin JR, Parks JH, Coe FL. Dependence of upper limit of metastability on supersaturation in nephrolithiasis. Kidney Int. 1997; 52:1602-1608,

4. Schinke T, Amendt C, Trindl A, et al. The serum protein alpha2HS glycoprotein/fetuin inhibits apatite formation in vitro and in mineralizing calvaria cells. A possible role in mineralization and calcium homeostasis. J Biol Chem. 1996; 271:20789-20796.
5. Schafer C, Heiss A, Schwarz A, et al. The serum protein alpha 2Heremans-Schmid glycoproteinfetuin-A is a systemically acting inhibitor of ectopic calcification. J Clin Invest. 2003; 112:357-366,

6. Heiss A, DuChesne A, Denecke B, et al. Structural basis of calcification inhibition by alpha 2-HS glycoproteinfetuin-A. Formation of colloidal calciprotein particles. J Biol Chem. 2003; 278:1333313341.

7. Westenfeld R, Schafer C, Kruger T, et al. Fetuin-A Protects against Atherosclerotic Calcification in CKD. J Am Soc Nephrol 2009; 20:1264-74

8. Schlieper G, Westenfeld R, Brandenburg V, Ketteler M. Inhibitors of calcification in blood and urine. Semin Dial. 2007; 20:113-21.

9. Stejskal D, Karpisek M, Vrtal R. et al. Urine fetuin-A values in relation to the presence of urolithiasis BJU International. 2008; 101:1151-1154.

10. Heiss A, Pipich V, Jahnen-Dechent W, Schwahn D. Fetuin-A is a mineral carrier protein: small angle neutron scattering provides new insight on Fetuin-A controlled calcification inhibition. Biophys J. 2010; 99:3986-95.

11. Zhou H, Pisitkun T, Aponte A, et al. Exosomal Fetuin-A identified by proteomics: A novel urinary biomarker for detecting acute kidney injury. Kidney Int. 2006; 70:1847-1857.

12.Price PA, Lim JE. The inhibition of calcium phosphate precipitation by Fetuin Is accompanied by the formation of a Fetuin-Mineral complex J Biol Chem. 2003; 278:22144-22152.

\section{Correspondence}

Ester Illiano, MD (Corresponding Author)

ester.illiano@inwind.it

Domenico Prezioso, MD

dprezioso@libero.it

Department of Gynecology, Obstetric and Urology,

University Federico II, Naples, Italy

Alberto Saita, MD

alsaurol@hotmail.com

Mario Motta, MD

mmotta@unict.it; mmotta@mbox.unict.it

Department of Urology "Ospedale Vittorio Emanuele",

University of Catania, Catania, Italy

Massimo Porena, MD

uropg@rdn.it

Carla Micheli, MD

carla.micheli@libero.it

Department of Urology and Andrology, Ospedale Santa Maria

della Misericordia, University of Perugia, Perugia, Italy

Dario Bruzzese, $M D$

dbruzzes@unina.it

Department of Preventive Medical Sciences,

University Federico II, Naples, Italy

Vincenzo Bisesti, MD

vincenzo.bisesti@libero.it

Domenico Russo, MD

domenicorusso51@hotmail.com

Department of Nephrology, University Federico II, Naples, Italy

Paolo Ferrari, MD

pferrari@hesperia.it

Department of Urology, Ospedale Hesperia, Modena, Italy

Tullio Lotti, MD

pferrari@hesperia.it

University Federico II, Naples, Italy 